\title{
The Triad of Cognition, Language, and Communication in Traumatic Brain Injury: A Correlational Study
}

\author{
Jeevendra Kumar Darshini ${ }^{1}$ Mohammed Afsar ${ }^{20}$ \\ Dhaval Shukla ${ }^{3}$ Jamuna Rajeswaran ${ }^{2}$ \\ ${ }^{1}$ Department of Speech Pathology and Audiology, National Institute \\ of Mental Health and Neurosciences (NIMHANS), Bengaluru, \\ Karnataka, India \\ ${ }^{2}$ Department of Clinical Psychology, National Institute of Mental \\ Health and Neurosciences (NIMHANS), Bengaluru, Karnataka, India \\ ${ }^{3}$ Department of Neurosurgery, National Institute of Mental Health \\ and Neurosciences (NIMHANS), Bengaluru, Karnataka, India \\ J Neurosci Rural Pract 2021;12:666-672.
}

Valiyaparambath Purushothaman Vandana ${ }^{1}$

Address for correspondence Valiyaparambath Purushothaman Vandana, PhD, Department of Speech Pathology and Audiology, National Institute of Mental Health and Neurosciences (NIMHANS), Bengaluru, Karnataka 560029, India (e-mail: vpvandana@gmail.com).

\section{Abstract \\ Keywords \\ - traumatic brain injury \\ - language \\ - cognition \\ - communication \\ - executive functioning}

Objectives Traumatic brain injury (TBI) is associated with deficits in cognition, language, and communication along with physical and psychosocial consequences. This study aimed at evaluating the association between cognition, language, and communication in a cohort of patients with TBI in southern India using standardized language specific assessment tools.

Materials and Methods The present correlational study included 30 patients with TBI of any severity, that is, mild, moderate, and severe within the postinjury period of 6 to 36 months. Comprehensive evaluation of cognition, language, and communication was done using National Institute of Mental Health and Neurosciences Neuropsychology Battery, Indian adapted versions of Western Aphasia Battery and La Trobe Communication Questionnaire (LCQ) respectively.

Data Analysis Measures of descriptive statistics, that is, mean, median, percentages, and frequencies, were used. Raw scores, that is, aphasia quotient (AQ), and subdomain scores for LCQ, that is, LCQ-quality, quantity, relation, manner, and cognition, were calculated. Composite scores for the three neuropsychological domains, that is, attention and processing speed, executive function, learning and memory, were evaluated. Partial correlation was computed for identifying the association between the three variables by controlling for age, education, duration, and severity of injury. Results A significant correlation was found between $A Q$ and measures of attention and executive functioning and all measures of LCQ except LCQ-manner. Further, a significant correlation was found between executive functioning and LCQ-total, LCQquantity, and LCQ cognition. No correlation was found between measures of attention and memory with communication. published online

September 20, 2021
DOI https://doi.org/ 10.1055/s-0041-1734008. ISSN 0976-3147.

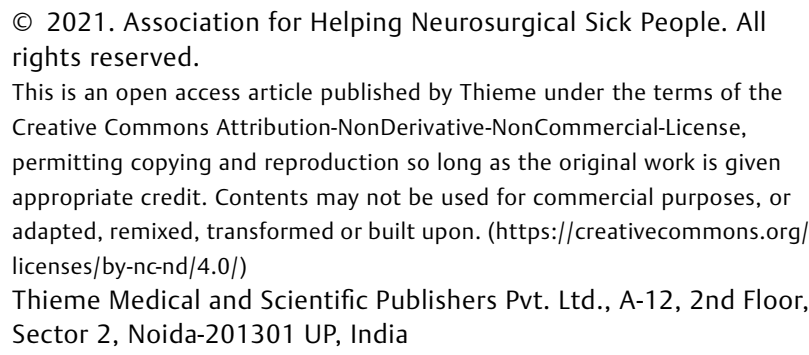

(C) 2021. Association for Helping Neurosurgical Sick People. All rights reserved.

This is an open access article published by Thieme under the terms of the Creative Commons Attribution-NonDerivative-NonCommercial-License, permitting copying and reproduction so long as the original work is given appropriate credit. Contents may not be used for commercial purposes, or adapted, remixed, transformed or built upon. (https://creativecommons.org/ licenses/by-nc-nd/4.0/)

Thieme Medical and Scientific Publishers Pvt. Ltd., A-12, 2nd Floor, Sector 2, Noida-201301 UP, India 
Conclusion A good language and executive functioning are associated with better communication abilities in patients with TBI thus emphasizing on the importance of a multidisciplinary team for the management of cognitive-communication impairments following a TBI.

\section{Introduction}

One of the complex clinical condition typically affecting the young adults is traumatic brain injury (TBI). It is associated with multiple long-term cognitive, physical, behavioral, and emotional consequences. ${ }^{1}$ Interpersonal functioning is also commonly affected following a TBI and is associated with poor long-term outcomes. ${ }^{2}$ At a larger level, TBI also affects the family members, society, and economy of a country as a whole. These posttraumatic consequences can further limit or prohibit the individual from returning to work after TBI and may affect the overall quality of life (QOL).

Verbal communication is unique to human beings and communication impairments following a TBI can be devastating. Communication impairments are found to persist for decades after the head injury. ${ }^{3}$ According to the American Speech and Hearing Association, difficulties with comprehension, rapidly spoken language, discourse, social language, abstract language, verbal learning and reasoning, communication in challenging environments, disinhibition, and imprecise language are some of the communication impairments reported following a TBI. ${ }^{4}$ Studies have also pointed toward the need to screen and evaluate individuals even with mild TBI for possible communication disorders. ${ }^{5,6}$

Language and cognition are the building blocks for effective communication and deficits in any aspects can affect an individual's overall communicative competence thus resulting in a communication breakdown. Language deficits are frequently reported following a TBI. Studies have revealed Broca's aphasia as the most frequent type of aphasia followed by anomic and transcortical motor aphasia following a TBI. ${ }^{7}$ Aphasia was found to persist in 13 patients of 39 individuals even after 15 years post-TBI. ${ }^{8}$ Deficits beyond the level of single words and sentences are reported in individuals postTBI, thus emphasizing that the traditional aphasia language batteries might be insufficient to explore the language impairments. ${ }^{9-11}$ It is usually the discourse that is tangential, disorganized, and even off-target when they are engaged in interaction. Thus, many studies have been conducted to evaluate the social aspects of language and they have revealed significant deficits in those with moderate-to-severe TBI. Inappropriate use of language and style of speech, taking greater numbers of turns in conversation, and involving their partners to a lesser extent leading to a difficulty in initiating and sustaining a meaningful conversation have been reported. ${ }^{12-14}$ Further, difficulties in organizing semantic content, producing fewer story grammar elements leading to incoherent and pragmatically inadequate discourse, are also reported in patients with TBI. ${ }^{15}$
The plethora of cognitive deficits commonly seen following a TBI include impaired attention and concentration, easy distractibility, impaired memory, impaired visuo-spatial abilities, slow information processing, poor judgement, and executive dysfunction. ${ }^{16}$ Studies have evaluated the neurocognitive consequences of TBI across all levels of severity. Sixty-five percent of individuals with moderateto-severe TBI have been found to have persistent cognitive deficits in long term, ${ }^{17}$ which in turn cause disability as they interfere with a patient's ability to perform their roles at work or home. Deficits in executive functioning, involving communication, lack of awareness of deficit, and intellectual ability, have also been reported in patients with TBI. ${ }^{18}$ Impairments in information processing speed and verbal memory were also found to be present 1-year after TBI resulting in chronic disability. The cognitive deficits after mild-to-moderate TBI are found to be resolved within 3 to 6 months postinjury. ${ }^{19-21}$ However, heterogeneity in TBI severity and patient characteristics can affect cognitive sequel thus complicating prediction of recovery.

Deficits in executive functioning also affect narrative discourse. There are three executive components that are involved in planning, monitoring, and execution of a message. These include-shifting, which involves the recall and integration of content for the story narrative; updating, which involves recall of past/previous episodes for the precise organization of a story; and inhibition, crucial for monitoring extraneous information and derailments during the course of story narration. ${ }^{22}$ Discourse depends on linguistic skills for grammatical processing, and to understand the communicative intentions of the speaker, it requires pragmatic processing. There is scientific evidence to suggest an association between executive functions and narrative skills in patients with TBI. ${ }^{14,15}$ This indicates the interrelationship between cognition and communication, and hence the term cognitive-communication disorder is used to explain the communication disorder in individuals with TBI due to cognitive deficits. ${ }^{23}$

It is increasingly becoming clear that deficits in cognition, language, and communication following TBI are interdependent. Although there are studies evaluating specific components of neuropsychological and communication deficits, there is a dearth of studies evaluating all the three domains simultaneously in this population. Further, the studies exploring the association between these domains in TBI population having a multilingual and culturally diverse background such as India are scarce. The cultural sensitivity and the unique characteristics of each language make it unjustified to import the findings from the studies done in 
the west. Using a standardized, valid, and language-specific test is crucial for accurate diagnosis of language disorders and a holistic evaluation of communication and cognition becomes equally essential for formulating appropriate rehabilitation plans for individuals with TBI. Thus, this study focused at evaluating the association between language, cognition, and communication in individuals with TBI in southern Indian peninsula using language specific test batteries.

\section{Methodology}

\section{Study Design}

This is a correlational study.

\section{Subjects}

Thirty participants in the age range of 25 to 55 years with diagnosis of TBI of any severity, that is, mild, moderate, or severe, were recruited from the outpatient's services of department of neurosurgery. The diagnosis was confirmed by a neurosurgeon. Right-handed English/Hindi/Kannada speaking individuals in the postinjury period of 6 to 36 months, with corrected vision and no hearing impairment was included in the study. Those with a past history of psychiatric, neurological, neurosurgical illness, substance use, or language disorder (e.g., global aphasia and severe dysarthria) were excluded.

\section{Procedure}

Participants were evaluated for language, communication, and cognitive functions. Language and communication assessment was conducted by a qualified speech-language pathologist (SLP) and the cognitive functioning was assessed by a qualified neuropsychologist. The testing was performed in two sessions, one each for the assessment of language and communication functions and the other for evaluation of cognition. Adequate rest periods between the sessions were provided to the participants to minimize fatigue effects. For the ethical reasons, all the participants and their family members were provided a feedback about the findings of assessment and adequate referrals were provided for rehabilitation where ever deemed necessary. The study complied with Helsinki Declaration and was accepted by the Institute Ethics Committee. Patients fulfilling study criteria were invited to participate in the study. Written informed consent was obtained from those willing to participate and their family members.

\section{Tools}

Western Aphasia Battery 24,25

Indian adaptation of the Western Aphasia Battery (WAB)Kannada and Hindi versions was used for the evaluation of language functions. The language skills evaluated include: spontaneous speech, repetition, auditory verbal comprehension, naming, reading, and writing. WAB provides aphasia quotient $(A Q)$ that provides the summary of overall severity of language impairment. Higher scores represent better language functioning.

\section{La Trobe Communication Questionnaire ${ }^{26}$}

La Trobe Communication Questionnaire (LCQ) was administered to evaluate the communicative functions. LCQ is a valid, standardized tool that was developed to evaluate the communicative abilities as perceived by the young adults having sustained a TBI and their relatives. ${ }^{26}$ It was developed based on the premise that the relatives have a knowledge of the patient's premorbid functioning and spend a lot of time with the patient and hence will be better to evaluate their communicative competence than the clinicians. It evaluates the behaviors for successful communication discourse that include quantity (using appropriate vocabulary, providing sufficient information, etc.), quality (providing accurate messages), manner (linguistic fluency, gaze efficiency, turn-taking, use of appropriate intonational contour), relation (topic maintenance, appropriate responses and speech style), and cognitive constructs (memory deficits, tangentiality, initiation difficulty, disinhibition, etc.). Higher scores represent poorer communication ability.

\section{NIMHANS Neuropsychology Battery ${ }^{27}$}

This battery consists of eight tests for the assessment of cognitive functioning. ${ }^{27}$ The cognitive domains evaluated include sustained attention, processing speed, working memory, verbal and visual learning, memory, semantic fluency, planning, and visuo-spatial construction. It is a standardized Indian battery that is widely used for clinical and research purposes.

\section{Data Analysis}

The statistical analyses were performed using the SPSS 20.0 for windows software. The raw scores from WAB and LCQ were directly used for analysis. The raw scores obtained from National Institute of Mental Health and Neurosciences (NIMHANS) neuropsychology battery were utilized to arrive at composite scores or indices for three domains, that is, attention and processing speed, executive functions, and learning and memory. Rohling's Interpretative Method for evaluating neuropsychological data was used to arrive at composite scores. ${ }^{28}$ These composite scores were used for further analysis. Higher composite scores represent better cognitive functioning.

The demographic and clinical profiles of individuals with TBI were described using measures of descriptive statistics, that is, frequencies, percentages, mean, and median. Partial correlation was computed for identifying the association between language, cognition, and communication measures with age, duration, education, and severity of injury controlled.

\section{Results}

Clinical and Sociodemographic Characteristics of the Participants

- Table 1 represents the clinical and sociodemographic characteristics of the participants. The age of the participants and years of education ranged from 20 to 56 years (mean age and SD: $35.9 \pm 8.5$ ) and 7 to 20 years (mean and SD: 
Table 1 Sociodemographic and clinical profile of the participants

\begin{tabular}{|l|l|}
\hline $\begin{array}{l}\text { Sociodemographic and } \\
\text { clinical variables }\end{array}$ & Mean \pm SD/n (\%) \\
\hline Age (y) & $35.9 \pm 8.5$ \\
\hline Years of education & $15.4 \pm 3.2$ \\
\hline Gender & \\
\hline Male & $26(87)$ \\
\hline Female & $4(13)$ \\
\hline Employment status post injury & \\
\hline Employed & $15(50)$ \\
\hline Unemployed & $15(50)$ \\
\hline Duration of illness in months & $14.36 \pm 9.95$ \\
\hline Injury severity & \\
\hline Mild & $06(20)$ \\
\hline Moderate & $17(57)$ \\
\hline Severe & $07(23)$ \\
\hline Presenting complaints & \\
\hline Memory and naming & $23(77)$ \\
\hline Language and speech & $7(23)$ \\
\hline
\end{tabular}

Abbreviation: SD, standard deviation.

$15.4 \pm 3.2)$, respectively. Most of the participants were males (87\%) and all the participants were gainfully employed prior to injury and $50 \%$ continued to be gainfully employed post injury.

The duration since injury ranged between 6 and 48 months (mean and SD: $14.36 \pm 9.95$ ) at the time of recruitment. All the participants had sustained road traffic accidents except for one who had sustained gunshot injury. Seventy-seven percent of the participants presented with disturbances in memory or slowness in thinking as their major complaints, while $23 \%$ presented with predominantly speech and language deficits.

\section{Language, Cognition, and Communication Variables}

The range, mean, and SD for the language, cognition, and communication variables are described in -Table 2. The language variable considered was AQ from WAB-Kannada and Hindi. The communication variables considered were the total score as well as domain scores of LCQ, that is, LCQquality, quantity, relation, manner and cognition. Attention processing speed index (APSI), executive functioning index (EFI), and learning and memory index (LMI) were the cognitive variables considered for the study.

\section{Association between Language and Cognition}

Language and cognitive functioning are prerequisites for effective verbal communication. Language and cognitive outcomes after TBI can be affected by age, education, duration since injury and injury severity and hence we initially established the partial correlation between the two with age,
Table 2 Descriptive statistics for the language, communication, and cognition variables

\begin{tabular}{|l|l|l|l|}
\hline & Range & Mean & $\begin{array}{l}\text { Standard } \\
\text { deviation }\end{array}$ \\
\hline \multicolumn{4}{|l|}{ Language variables } \\
\hline AQ & $33-99$ & 82.87 & 15.82 \\
\hline Communication variables \\
\hline LCQ-quality & $5-17$ & 6.03 & 0.96 \\
\hline LCQ-quantity & $4-8$ & 11.16 & 2.66 \\
\hline LCQ-relation & $6-12$ & 9.40 & 1.61 \\
\hline LCQ-manner & $13-21$ & 17.06 & 2.06 \\
\hline LCQ-cognition & $14-34$ & 24.80 & 4.11 \\
\hline LCQ-total & $45-86$ & 66.27 & 8.80 \\
\hline Cognitive variables & & & \\
\hline APSI & $0.26-0.95$ & 0.67 & 0.21 \\
\hline EFI & $0.00-0.85$ & 0.54 & 0.20 \\
\hline LMI & $0.00-0.94$ & 0.45 & 0.27 \\
\hline
\end{tabular}

Abbreviations: APSI, attention processing speed index; AQ, Aphasia quotient; EFI, executive function index; LCQ, La Trobe communication questionnaire; LMI, learning and memory index.

duration since injury, education, and severity of head injury controlled. The correlation coefficients and $p$-values for the language and cognitive variables are described in - Table 3. Results revealed that AQ correlated moderately with EFI and LMI but no significant correlation was found between AQ and APSI. These results suggest that better language functioning is associated with better executive functioning and memory.

\section{Association of Communication with Language and Cognition}

Next, we attempted to analyze the association of communication variables with language and cognition. - Table 4 presents the partial correlations of LCQ scores with AQ, APSI, EFI, and LMI, with age, education, duration since the injury, and severity of head injury controlled.

- Table 4 shows that AQ is significantly correlated with LCQ-total and all sub-domains of LCQ except LCQ-Manner. These findings indicate that better language functioning is associated with better communication. APSI and LMI are not

Table 3 Correlation between language functioning and various domains of cognitive functioning (age, education, duration, and severity of injury controlled)

\begin{tabular}{|l|l|l|l|}
\hline & APSI & EFI & LMI \\
\hline AQ & 0.28 & $0.62^{\mathrm{a}}$ & $0.54^{\mathrm{b}}$ \\
\hline
\end{tabular}

Abbreviations: APSI, attention processing speed index; AQ, Aphasia quotient; EFI, executive function index; LCQ, La Trobe Communication Questionnaire; LMI, learning and memory index.

a $p$-Value significant at 0.01 .

${ }^{\mathrm{b}} \mathrm{p}$-Value significant at 0.05 . 
Table 4 Correlation of communication ability with language functioning and various domains of cognitive functioning (age, education, duration and severity of injury controlled)

\begin{tabular}{|l|l|l|l|l|}
\hline & AQ & APSI & EFI & LMI \\
\hline LCQ-total & $-0.71^{\mathrm{a}}$ & -0.26 & $-0.55^{\mathrm{b}}$ & -0.35 \\
\hline LCQ-quantity & $-0.73^{\mathrm{a}}$ & -0.31 & $-0.58^{\mathrm{a}}$ & -0.36 \\
\hline LCQ-quality & $-0.44^{\mathrm{b}}$ & 0.06 & -0.02 & 0.01 \\
\hline LCQ-relation & $-0.46^{\mathrm{b}}$ & -0.07 & -0.43 & -0.32 \\
\hline LCQ-manner & -0.32 & -0.35 & -0.19 & -0.09 \\
\hline LCQ-cognition & $-0.63^{\mathrm{a}}$ & -0.19 & $-0.60^{\mathrm{a}}$ & -0.39 \\
\hline
\end{tabular}

Abbreviations: APSI, attention processing speed index; AQ, Aphasia quotient; EFI, executive function index; LCQ, La Trobe Communication Questionnaire; LMI, learning and memory index.

a $p$-Value significant at 0.01 .

${ }^{\mathrm{b}} \mathrm{p}$-Value significant at 0.05 .

significantly correlated with any of the LCQ scores. However, EFI has significant correlation with LCQ-total, LCQ-quantity, and LCQ-cognition indicating that better executive functioning is associated with better communication ability.

\section{Discussion}

The present study aimed at evaluating the association between language, cognition, and communication following TBI using comprehensive and standardized assessment tools. Since language and cognition form the basis for communication, we first attempted to establish the association between the two and found that language abilities as measured by AQ were significantly associated with executive functions and memory. The association between language and executive functions is in line with existing literature. A recent study established that in children the receptive language was associated with nonverbal working memory, whereas syntactic abilities were predicted by nonverbal inhibition. ${ }^{29}$ This association seems to be supported by neuroanatomical basis as there is scientific evidence which points that language processing and executive functions are mediated by a partially shared network consisting of frontal, parietal, and subcortical areas of the brain. ${ }^{30}$ The significant association between language and memory functions can be understood in the light of the nature of the tests used to assess memory. The LMI was calculated using the raw scores from Rey's Auditory Verbal Learning Test (RAVLT) and Complex Figure Test (CFT). RAVLT requires the participant to say aloud the words read to him orally thus relying on lexical memory, a major aspect of language. In CFT, the participant has to copy a complex design first by copying and later by memory. Though CFT is traditionally considered to be a nonverbal test, there is evidence suggesting that nonverbal material may be encoded using verbal codes. ${ }^{31}$ Given highly verbal nature of the tests used to assess the memory functions, it is not surprising to see a significant correlation between language and memory functions. Further, a successful performance on both RAVLT and CFT requires good executive control in the form of organization of material and strategy to recall. Therefore, the relationship between the memory and language may be mediated by executive functions. With regard to the specific component of language, auditory verbal comprehension has been found to be a significant predictor for cognitive outcomes in individuals with aphasia post-TBI ${ }^{32}$ that further highlights the relationship between language and cognition.

A dissociation in the studies exploring basic language functions in patients with TBI on one hand and communication on the other was identified in the literature review. We addressed this lacuna by studying the association between these two aspects. A significant association between AQ with the overall measure of communication derived from LCQ and with the quantity, quality, relation, and cognition aspects of LCQ was found. This is in consensus with the profile of language and communication deficits reported following TBI that include word-retrieval deficits, disorganized and tangential oral or written discourse, difficulty in communicating in stressful environments, deficits in comprehension of oral or written language, and changing the interaction style according to the situation. ${ }^{4}$

Further, we found that only the executive functions domain of cognitive functioning had a significant association with LCQ total, LCQ-quantity, and LCQ-cognition. Other domains of cognitive functioning did not have significant association with measures of communication. This selective association of executive functions with communication can be understood in the light of the nature of items in the tool used to assess the communication. The items in LCQ were selected based on the description of cognitive-communication deficits following TBI. ${ }^{32}$ Further, most of the studies have investigated the role of executive functioning on communication by specifically evaluating the communicative discourse. Studies have revealed that discourse impairment reflects dysfunction in executive control over cognitive and linguistic organizational processes, regardless of lesion type or location. ${ }^{33,34}$ Significant correlations have also been established between scores on tasks evaluating executive functioning with that of story grammar, which is a task for superstructural measure of discourse in individuals with TBI. $^{35}$

It is noteworthy that overall communication ability, along with the quantity and cognition aspects of communication, is associated with both language and executive functioning. However, the quality and relation aspects of communication are only associated with language and not with executive functioning. These findings suggest that communication breakdown due to poor 'quantity' and 'cognition' can be associated with either executive function and/or language deficits. However, communication impairments due to poor "quality" that is related to the accuracy of contribution and "relation" that is related to the relevance of contribution are mainly associated with language deficits. This specific pattern of association of various aspects of communication ability can help the clinicians to understand the factors underlying the communicative incompetence. Patient with communication breakdown secondary to poor quantity and 
cognition aspects may require both speech-language and neuropsychological rehabilitation. On the other hand, patients with communication breakdown secondary to quality and relation aspects may only require speech-language rehabilitation.

The findings from the present study need to be interpreted with caution owing to the limitations of the study that include smaller sample size and mixed severity of the participants recruited. Prospective studies with a larger sample size can enhance the generalizability of the findings of the study. Although bilingualism is an inherent part of the people in India, the participants were only evaluated in their proficient language and were not grouped on the basis of bilingualism, which can have an impact on their cognition and linguistic abilities.

Based on our knowledge, this is the first study in the Indian context to evaluate the association between language, cognition, and communication skills. Such a study can better inform the SLPs and neuropsychologists involved in the assessment and management of cognitive-communication deficits following a TBI in planning and providing appropriate intervention and further contribute to improvement in the overall QOL.

\section{Conclusion}

This study investigated the association between cognition, language, and communication in patients with TBI. The findings have revealed a significant association between measures of language and executive functioning with communication. This provides an insight to the SLPs and neuropsychologists about the various components of language and cognition that influence communication as a whole thus emphasizing on the need for a multidisciplinary team for the intervention of cognitive-communication impairments following a TBI.

\section{Authors' Contributions}

D.J.K. was involved in design and acquisition of data, analysis and interpretation of data, drafting of the article, and the critical revision of the manuscript; M.A. was involved in design and acquisition of data, analysis and interpretation of data, drafting of the article, and the critical revision of the manuscript; V.V.P. was involved in conception and design, drafting of the article, and the critical revision of the manuscript for important intellectual content; D.S. was involved in design, data interpretation, revision of the manuscript critically for important intellectual content; J.R. was involved in the critical revision of the manuscript for intellectual content. All authors read and approved the final manuscript.

\section{Ethical Approval}

The study is in compliance with the Declaration of Helsinki and was approved by the Research Ethics Committees of NIMHANS, Bengaluru. Informed consent was obtained from all the participants and their family caregivers.

\section{Funding}

The study was funded by the Defence Research \& Development Organization-Life Sciences Research Board (DRDO-LSRB), Government of India (Grant reference No: O/o DG(TM)/81/48222/LSRB-309).

\section{Conflict of Interest}

None declared.

\section{References}

1 De Lisa JA, Gans BM, Walsh NE. Physical Medicine and Rehabilitation: Principles and Practice. 4th edition. Philadelphia: Lippincott Williams \& Wilkins; 2005

2 Togher L. Cognitive communication disorders after traumatic brain injury. In: Guendouzi J, Loncke F, Williams MJ, eds. The Handbook of Psycholinguistic and Cognitive Processes: Perspectives in Communication Disorders. New York: Psychology Press; 2011:603-624

3 Raymont V, Salazar AM, Krueger F, Grafman J. "Studying injured minds" - the Vietnam head injury study and 40 years of brain injury research. Front Neurol 2011;2:15

4 Position statement: The role of speech-language pathologists in the identification, diagnosis, and treatment of individuals with cognitive-communicative impairments. ASHA 1988;30(03):79

5 Norman RS, Jaramillo CA, Amuan M, Wells MA, Eapen BC, Pugh MJ. Traumatic brain injury in veterans of the wars in Iraq and Afghanistan: communication disorders stratified by severity of brain injury. Brain Inj 2013;27(13-14):1623-1630

6 Krug H, Turkstra LS. Assessment of cognitive-communication disorders in adults with mild traumatic brain injury. Perspect Neurophysiol Neurogenic Speech Lang Disord 2015;25(01): 17-35

7 Ozbudak Demir S, Görgülü G, Köseoglu F. Comparison of rehabilitation outcome in patients with aphasic and non-aphasic traumatic brain injury. J Rehabil Med 2006;38(01):68-71

8 Ludlow CL, Rosenberg J, Fair C, Buck D, Schesselman S, Salazar A. Brain lesions associated with nonfluent aphasia fifteen years following penetrating head injury. Brain 1986;109(Pt 1):55-80

9 Biddle KR, McCabe A, Bliss LS. Narrative skills following traumatic brain injury in children and adults. J Commun Disord 1996;29 (06):447-468, quiz 468-469

10 Hux K, Wallace S, Evans K, Snell J. Performing cookie theft picture content analyses to delineate cognitive-communication impairments. J Med Speech-Lang Pathol 2008;16(02):83-99

11 Coelho CA. Discourse production deficits following traumatic brain injury: A critical review of the recent literature. Aphasiology 1995;9(05):409-429

12 Ylvisaker M. Communication outcome following traumatic brain injury. Semin Speech Lang 1992;13(04):239-251

13 Marsh NV, Knight RG. Behavioral assessment of social competence following severe head injury. J Clin Exp Neuropsychol 1991;13 (05):729-740

14 Coelho CA, Liles BZ, Duffy RJ. Analysis of conversational discourse in head-injured adults. J Head Trauma Rehabil 1991;6(02):92-99

15 Miyake A, Friedman NP, Emerson MJ, Witzki AH, Howerter A, Wager TD. The unity and diversity of executive functions and their contributions to complex "Frontal Lobe" tasks: a latent variable analysis. Cognit Psychol 2000;41(01):49-100

16 Arciniegas DB, Held K, Wagner P. Cognitive impairment following traumatic brain injury. Curr Treat Options Neurol 2002;4(01): 43-57

17 Whiteneck GG, Gerhart KA, Cusick CP. Identifying environmental factors that influence the outcomes of people with traumatic brain injury. J Head Trauma Rehabil 2004;19(03):191-204 
672 The Triad of Cognition, Language, and Communication in TBI Darshini et al.

18 Ruff RM, Cullum CM, Luerssen TG. In: Bigler ED, Yeo RA, Turkheimer E, eds. Neuropsychological Function and Brain Imaging. Critical Issues in Neuropsychology. Boston: Springer; 1989:161-162

19 Carroll LJ, Cassidy JD, Peloso PM, et al; WHO Collaborating Centre Task Force on Mild Traumatic Brain Injury. Prognosis for mild traumatic brain injury: results of the WHO collaborating centre task force on mild traumatic brain injury. J Rehabil Med 2004;36 (43, Suppl):84-105

20 Frencham KA, Fox AM, Maybery MT. Neuropsychological studies of mild traumatic brain injury: a meta-analytic review of research since 1995. J Clin Exp Neuropsychol 2005;27(03):334-351

21 Schretlen DJ, Shapiro AM. A quantitative review of the effects of traumatic brain injury on cognitive functioning. Int Rev Psychiatry 2003;15(04):341-349

22 Mozeiko J, Le K, Coelho C, Krueger F, Grafman J. The relationship of story grammar and executive function following TBI. Aphasiology 2011;25(6-7):826-835

23 College of Audiologists and Speech-Language Pathologists of Ontario. Preferred Practice Guideline for Cognitive-Communication Disorders. Ontario, Canada: College of Audiologists and Speech-Language Pathologists of Ontario; 2002

24 Kertesz A. Western Aphasia Battery Test. New York: The Psychological Corporation; Harcourt, Brace, Javanovich, Inc.; 1982

25 Karanth P. Western Aphasia Battery in Hindi. Mysore: ICMR Project, All India Institute of Speech and Hearing; 1980

26 Douglas JM, O'Flaherty CA, Snow PC. Measuring perception of communicative ability: the development and evaluation of the La Trobe Communication Questionnaire. Aphasiology 2000;14(03): 251-268
27 Rao SL, Subbakrishna DK, Gopukumar K. NIMHANS neuropsychology battery. Bangalore: National Institute of Mental Health and Neurosciences; 2004

28 Miller LS, Rohling ML. A statistical interpretive method for neuropsychological test data. Neuropsychol Rev 2001;11(03): 143-169

29 Kaushanskaya M, Park JS, Gangopadhyay I, Davidson MM, Weismer SE. The relationship between executive functions and language abilities in children: A latent variables approach. J Speech Lang Hear Res 2017;60(04):912-923

$30 \mathrm{Ye} \mathrm{Z,} \mathrm{Zhou} \mathrm{X.} \mathrm{Executive} \mathrm{control} \mathrm{in} \mathrm{language} \mathrm{processing.} \mathrm{Neurosci}$ Biobehav Rev 2009;33(08):1168-1177

31 Tversky B. Pictorial and verbal encoding in a short-term memory task. Percept Psychophys 1969;6(04):225-233

32 Demir SO, Altinok N, Aydin G, Köseoğlu F. Functional and cognitive progress in aphasic patients with traumatic brain injury during post-acute phase. Brain Inj 2006;20(13-14): 1383-1390

33 Coelho CA, Liles BZ, Duffy RJ. Discourse analyses with closed head injured adults: Evidence for differing patterns of deficits. Arch Phys Med Rehabil 1991;72(07):465-468

34 Ylvisaker M, Szekeres SF, Feeney T. Communication disorders associated with traumatic brain injury. In: Chapey R, ed. Language Intervention Strategies in Aphasia and Related Neurogenic Communication Disorders. Baltimore, MD: Williams and Wilkins; 2001:745-800

35 Coelho CA, Liles BZ, Duffy RJ. Impairments of discourse abilities and executive functions in traumatically brain-injured adults. Brain Inj 1995;9(05):471-477 